PROKLA-Redaktion

\section{Editorial: Umkämpfte Arbeit}

Arbeit ist in Deutschland wieder ins Zentrum gesellschaftlicher und politischer Auseinandersetzungen gerückt. Die Debatte um Mindestlöhne, der Streik der Lokomotivführer, die Konflikte um die Umsetzung des Entgeltrahmenabkommens in der Metallindustrie, die Proteste gegen die geplant Schließ all dies zeigt, dass sich die Enwerbsarbeit und ihre Einbindung in nicht erwerbsförmige Arbeit in den letzten beiden Jahrzehnten weitreichend verändert haben, damit aber auch die Formen und die Ziele der Kämpfe urn sie.

$\mathrm{Da}$ ist zum einen die zunehmende Erosion des tarifrechtlichen Schutzes. Nicht nur die Kräfteverhältnisse in den Unternehmen haben sich verschoben, sondern diese Entwicklung wurde zudem von einer Politik zugunsten der Interessen der Kapitalanleger wohlwollend begleitet. Unisono forderten „Wirtschaft" und „Politik“", das Gebot der "Flexibilität" in der Arbeit durch zusetzen und die Aushandlung der Entlohnung auf die Unternehmensebene zurückzuführen. Flankiert und gefördert von Veränderungen im Arbeits- und Sozial recht mutierten die Flächentarifverträge daher immer stärker zu einem mehr oder weniger lockeren Rahmen für betrieblich ausgehandelte Sonderregelungen. Daneben sind erhebliche tarifvertragsfreie oder von „gelben Gewerkschaften“ im Interesse der Unternehmensführungen vertretene Zonen entstanden, und zwar vor allem im Niedriglohnbereich.

Dies hat nicht nur die Arbeits- und Lebensbedingungen der meisten Lohnabhängigen verschlechtert, sondern schließlich auch eine wichtige Funktion kollekti- ver Regelungen fuir die Kapitalseite unterminiert - die Dämpfung der Konkurrenz durch Lohn- und Arbeitszeitstandards. Die Kämpfe um einen Mindestlohn haben daher mindestens zwei Seiten. Von gewerkschaftlicher Seite handelt es sich um Versuche, die Politik ins Spiel zu bringen, um die schwächsten Kategorien der Lohnabhängigen vor einem freien Fall ihrer Einkommen $\mathrm{zu}$ schützen. In bestimmten Branchen scheint es aber auch ein zunehmendes Interesse an einer Schadensbegrenzun durch Teile des Kapitals zu geben, die in der verschärften Unternehmenskonkurrenz zu Verlierern zu werden drohen.

Zum anderen stellt sich das politische Problem, die Einheit der Lohnabhängigen bzw. der subalternen Gruppen gegen Ausbeutung und Herrschaft herzustellen, auf neue Weise, weil sich die Formen ihre Differenzierung verändert haben. So sind in den letzten Jahrzehnten z.B. neue Tätigkeits- und Berufskategorien entstanden, die eher auf andere Formen der Vernetzung als die gewerkschaftliche setzen, um mit den Unsicherheiten ihrer Arbeitsmöglichkeiten und -bedingungen fertig zu werden. Dazu gehört die wachsende Gruppe der zwischen Lohnabhängigkeit und Selbständigkeit angesiedelten ,Alleinselbständigen". Da sich die Zugriffsmöglichkeiten und Zugriffsweisen der Unternehmen auf die Arbeitskräfte verändert haben, nehmen auch die Konflikte um Lohn und Leistung neue Formen an und verbinden sich auf neue Weise mit Fragen der Kontrolle über die Arbeit und ihre Ergebnisse.

Nach dem Ende des Zweiten Weltkriegs haben sich in der Bundesrepublik Verhältnisse herausgebildet, die später vielfach als „fordistisch" bezeichnet wurden. Gemeint war damit die Durchsetzung tayloristischer schreitender Mechanisierung und Bürokratisierung des Arbeitsprozesses. Konzepte dieser Art gingen in erster Linie auf dic USA zurück, wo Frederick Taylor bereits vor dem Ersten Weltkrieg hoffte, mit Hilfe seiner „wissenschaftlichen Betriebsfüh rung" den Eigensinn der Arbeiterschaft zu brechen und sie mit Hilfe von autoritativer Anleitung und Lohnanreizen auf die Ziele des Kapitals zu verpflichten. Mit er heblich größerem praktischen Erfolg als Taylor setzte Henry Ford, der im übrigen jeden Verweis auf Taylor peinlichst ver mied, das Konzept der strikten Trennung zwischen planender und ausführender Arbeit in die industrielle Realität um und ergänzte es um Spezialmaschinen und Fließbänder. Seine Arbeiter wurden somit dem Tempo des Fließbands bzw. des Maschinensystems unterworfen - mit dem Ergebnis einer ungeheuren Intensivierung der Arbeit, aber auch gewaltiger Produktivitätssteigerungen. Diese wurden zum Teil dazu genutzt, die produzierten Automobile zu niedrigeren Preisen und damit auch an die Arbeiter zu verkaufen. Die Arbeitsbedingungen der mechanisierten Massenproduktion - meisterhaft karikiert in Charlie Chaplins Modern Times - wurden von der Arbeiterschaft jedoch erst dann akzeptiert, als das Unternehmen erhebliche Lohnerhöhungen zugestand. Der legendäre Five-Dollar-Day schränkte die starke (und für das Unternehmen extrem teure) Fluktuation ein, war allerdings auch mit der Anforderung unbedingter Loyalität gegenüber dem Arbeitgeber verbunden.

Was Henry Ford selbst grandios als „WeiBen Sozialismus" verkündete, wurde nicht nur von deutschen Unternehmern, sondern selbst von Gewerkschaftlem der Zwischenkriegszeit ehrfürchtig bestaunt schien doch hier ein Gesellschaftsmodell heraufzudämmern, das eine Versöhnung von Arbeit und Kapital und somit ein Ende der unseligen Klassenkämpfe versprach. Kritisierte Lenin den Taylorismus zunächst als „Schweißauspressung nach al- len Regeln der Wissenschaft" (LW 18, 589) und „Versklavung des Menschen durch die

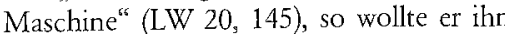
später selbst zum Aufbau des Sozialismus cinsetzen (vgl. LW 27, 249f; LW 33, 354f) In Deutschland wurden in den 1920er und 1930er Jahren ganze Bibliotheken mit Literatur zur Rationalisierung gefüllt, Taylors und Fords Konzepte jedoch nur ansatzweise verwirklicht. Die durchschlagendste Neuerung war die Einfuihrung des REFAMannes, der die Akkorde mit der Stoppuhr in der Hand festlegte, während dies bis dahin die Meister nach Gefühl und Erfahrung gemacht hatten. (Das bis heute gebräuchliche Akronym REFA bezeichnet den Reichsausschuss für Arbeitszeitermitt lung - eine der zahllosen Kommissionen, die noch während des Ersten Weltkriegs gegründet worden waren, um die Ziele der Rationalisierung voranzubringen).

Der Siegeszug des tayloristisch-fordistischen Produktionsmodells setzte in Westdeutschland mit den 1950er Jahren ein und ebenso wie in den USA fand er sein ideales Terrain in der Automobilindustrie vor: Der VW-Käfer, eine Konstruktion Ferdinand Porsches und ein Lieblingsprojekt Adolf Hitlers, trat während des Krieges zwar lediglich als „Kübelwagen" in Erscheinung, wurde aber dann in der Bundesrepublik zum Inbegriff des Autos für den "kleinen Mann". Nun erst griff das fordistische Konzept, standardisierte Produkte in großen Serien herzustellen, sol cherart die Produktionskosten zu senken und der Arbeiterschaft über gestiegene Löhne den Kauf dieser Produkte zu ermöglichen (bis dahin hatte dies allenfalls für Rundfunkgeräte funktioniert). Im gewaltigen Aufschwung der Jahre des westdeutschen "Wirtschaftswunders" konnte das Produktionsmodell auch auf andere Gegenstände des täglichen Bedarfs ausgeweitet werden. So kam die deutsche Hausfrau zu ihrer ersten Constructa-Vollwaschmaschine, die Familie versammelte sich mit den Nachbarn vor dem SchwarzweißFernseher von Grundig oder ging aus, um im Wienerwald die ersten massenproduzierten Hühner zu verspeisen. 
Was es bedeutet, die ersten drei Jahrzehnte der Bundesrepublik als „fordistisch“ zu bezeichnen, bleibt dennoch umstritten. Bei näherem Hinsehen zeigt sich, dass die Landschaft des damaligen Arbeitslebens doch wesentlich vielfältiger war. So gab es Bereiche, in denen zwar eine tayloristische Arbeitsteilung und somit monotone und belastende Arbeitsbedingungen vorherrschten, diese aber keine Kompensation in relativ hohen Löhnen fanden - und zwar dann, wenn nicht Arbeiter, sondern Arbeiterinnen das Gros der Arbeitskräfte stellten, wie bei vielen Montagetätigkeiten in der Elektroindustrie oder in dem (damals noch weit verbreiteten) Textil- und Bekieidungsgewerbe. Im Übrigen war die Degradierung der männlichen Arbeiter selbst in der Automobilindustrie nie so umfassend, wie es Ford in seinem River Rouge-Werk vorgemacht hatte, wo die ganz große Mehrzahl aller Tätigkeiten angelernt oder ungelernt war. In der Bundesrepublik existierte demgegenüber - auch bei den Unternehmern - traditionell eine Hochschätzung der Facharbeit, an deren Ausgestaltung im Rahmen des dualen Systems der Berufsausbildung Arbeitgeber wie Gewerkschaften mitwirkten.

In den Industrien, die standardisierte Massengüter herstellten, behielten die Gelernten daher erhebliche Bedeutung, und um so mehr galt dies in solchen, für die kleine Serien, Auftragsfertigung und stetige Innovationen typisch waren, wie in Teilen des Maschinenbaus. Im Übrigen war ohnehin nur eine Minderheit aller Arbeitskräfte in den Fabriken der großen Unternehmen wie Volkswagen, Ford, Opel, Siemens, Bosch oder Mannesmann beschäftigt. Für die Mehrheit fand der Arbeitsalltag in kleinen und mittelgroßen Unternehmen statt, für viele davon in handwerklichen Betrieben, deren Bedeutung bis zum heutigen Tag kaum abgenommen hat. Der beliebteste Ausbildungsberuf für junge Männer blieb über Jahrzehnte hinweg derjenige des KfZ-Mechanikers.

Dennoch wurden die Verhältnisse in den großen Unternehmen prägend für die allermeisten Beschäftigten. Denn dort wur- den insbesondere von der IG Metall als Vorreiterin Verbesserungen durchgesetzt, die nach und nach zum allgemeinen Standard wurden: insbesondere regelmäßige Tarifverhandlungen, über die die Löhne im Großen und Ganzen an die Produktivitätsfortschritte gekoppeli wurden, und Verkürzungen der täglichen und der wöchentlichen Arbeitszeit. Einer der erfolgreichen IG Metall-Slogans dieser Zeit lautete: "Samstags gehört Vati mir". Dazu kam - über lange Jahre unter der Ägide konservativer Regierungen - eine sich ausweitende sozialstaatliche Absicherung, darunter die Dynamisierung der Renten, der Ausbau der verschiedenen Zweige der Sozialversicherung und die Einführung der Sozialhilfe als Rechtsanspruch.

Kennzeichnend für diese Periode war somit die Herausbildung eines "Normalarbeitsverhältnisses", das allerdings erst dann so bezeichnet wurde, als es für viele, vor allem für die Berufseinsteiger/innen, gerade nicht mehr selbstverständlich war. Typisch dafür waren die unbefristete Vollzeitbeschäftigung mit gesetzlich und kollektivvertraglich geregelten Arbeitszeiten, steigendem Lohn sowie Absicherungen für Krankheiten, Unfälle, Invalidität, Arbeitslosigkeit und Alter. Nicht alle Beschäftigen waren gleichermaßen Nutznießer dieses Arrangements: erwerbstärige Männer mehr als erwerbstätige Frauen, Qualifizierte mehr als An- und Ungelernte, kontinuierlich Beschäftigte mehr als Arbeitskräfte mit Brüchen in ihrer Biographie, Inländer mit beruflichen Aufstiegschancen mehr als Migranten in beruflichen Sackgassen.

Was vielen heute als goldene Ära eines endgültig krisenfreien und sozial ausgewogenen Kapitalismus vorkommt, wies be reits Ende der $1960 \mathrm{er} \mathrm{Jahre} \mathrm{erste} \mathrm{Risse} \mathrm{auf.}$ Die Produktivitätszuwächse, die auf der Basis der tayloristisch-fordistischen Arbeitsorganisation erzielt wurden, waren rückläufig da der Arbeitsprozess sich über einen bestimmten Punkt hinaus nicht weiter zerstückeln ließ. Auch die Intensivierung der Arbeit stieß an Grenzen und die Kosten für die fortschreitende Mechanisierung des Arbeitsprozesses nahmen zu. Überdies kam es zu (wilden) Streiks, Absentismus und Sabotage, also zu Widerstandsaktionen der Arbeiterschaft, deren Möglichkeit man mittlerweile schon fast vergessen hatte und die als überholt und einer anderen Zeit zugehörig schienen. Häufig waren gerade Migranten, die sogenannten "Gastarbeiter", an ihnen beteiligt. Im Ergebnis erreichten sie noch einmal erhebliche Lohnsteigerungen, einen weiteren Ausbau de Sozialstaats (nunmehr unter sozialliberalen Vorzeichen), eine faktische Verstetigung des Aufenthalts der migrantischen Arbeiter/innen und liberalere Regeln des Familienzuzugs. Es sah aus, als säße die Arbeiterschaft am längeren Hebel.

Den Unternehmen drohte jedoch auch von anderer Seite Ungemach, da sich die wirtschaftlichen Machtpositionen zwischen den kapitalistischen Zentren zunehmend verschoben. So wurde mit der nachholenden Entwicklung in Westeuropa und Japan und dem Krieg in Vietnam die bis dahin unbestrittene Hegemonie der USA fraglich, dazu kam aber auch der unübersehbare Aufstieg mehrerer "Schwellenländer", die man nicht mehr einer weit abgeschlagenen „Dritten Welt" zurechnen konnte, sondern die, wie die asiatischen "Tigerstaaten", zunehmend zu industriellen Konkurrenten der europäischen Länder und der USA wurden. Für besonders lohnintensive Industriezweige wie dic deutsche Textil- und Bekleidungsindustric wurde es lohnend, bestimmte Fertigungsschritte in "Billiglohnländer" auszulagern. Im Zuge der sich verschärfenden weltweiten Konkurrenz bildeten sich in vielen Bereichen Überkapazitäten heraus.

Durch den Rückgang der Produktivitätszuwächse verengte sich zum einen der Verteilungsspielraum zwischen den Klassen. Zum anderen blieben die Produktivitätszuwächse im Verhältnis zu den mittlerweile mäßigen Wachstumsraten des Sozialprodukts jedoch so hoch, dass eine wachsende strukturelle Arbeitslosigkeit entstand. Diese neuartige Situation verwies die bisher selbstbewussten Gewerkschaften in die Defensive, da sich das Kräftever hältnis zwischen Arbeit und Kapital seit
Mitte der 1970er Jahre deutlich zu Ungunsten der Lohnabhängigen verschob. Dadurch wurden schließlich auch die Voraussetzungen für den Übergang vom zunächst noch eher keynesianisch geprägten Krisenmanagement zu neoliberalen Konzepten geschaffen. Unter dem Druck der Hochzinspolitik sahen sich die Unternehmen gezwungen, die Restrukturierung der Produktion voranzutreiben. Dabei wurden freilich sehr unterschiedliche Wege beschritten.

Zum einen ergab sich mit der Entwicklung der Mikroelektronik die Möglichkeit, die Automation zu forcieren. Die Vision von der menschenleeren Fabrik erwies sich jedoch schnell als Illusion. In den 1980er Jahren entstanden sprichwörtliche „Ruinen" des Computer Integrated Manufacturing (CIM). Gleichwohl hat der Einsatz der Computer die Arbeitsprozesse in vie len Bereichen umgewälzt, insbesondere be den white collar- oder Angestelltenarbeiten, also bei Forschung und Entwicklung, in der Verwaltung und in der Distribution, wo Prozesse der Rationalisierung in den Jahren zuvor eher marginal geblieben waren. Mit Hilfe von Informations- und Kommunikations-Technologien wurde die Büroarbeit nun sehr viel stärker normiert, kieinräumiger kontrolliert und intensiviert, teilweise auch in Fließprozesse umgewandelt oder auslagerbar gemacht. Dic schrittweise Verallgemeinerung und Verbitligung der Computertechnologie bedeutete eine "Verwohlfeilerung der Elemente des konstanten Kapitals" (Marx), die zur Wiederherstellung der Profitabilität des Kapitals beitrug.

Die andere Antwort auf die Krise bestand in Experimenten mit einer veränderten Arbeitsorganisation. Teils reagierten die Unternehmer damit auf den Widerstand der „Massenarbeiter" und erwarteten gleichzeitig Produktivitätssteigerungen, teils gab es gewerkschafflich initiierte arbeitspolitische Strategien der „Humanisierung der Arbeit", bei denen die Vertreter der Arbeiterschaft hofften, an ihre früheren Erfolge anzuknüpfen, um auch gegen den Widerstand des Kapitals Alternativen 
zum Taylorismus durchzusetzen. So kamen Maßnahmen wie job enrichment, job enlargement, teilautonome Arbeitsgruppen und andere auf die Tagesordnung. Als Höhepunkt dieser Entwicklung kann da in den 1980er Jahren geplante Montagewerk von Volvo in Uddevalla gelten, in dem das Fließsband abgeschafft und durch die quasi „handwerkliche ${ }^{6 s}$ Fertigung von Fahrzeugen durch kleine Teams in Montagewerkstätten ersetzt wurde.

Seit Ende der 1980er Jahre wurden in verschiedenen Ländern, aber auch in einzelnen Unternehmen durchaus unterschiedliche Pfade der Restrukturierung der Arbeit beschritten, die sich in einem Spannungsverhältnis von defensiver und offensive Flexibilisierung, von Neotaylorismus und Formen der ,ausgehandelten Einbindung und ,verantwortlichen Autonomie" der Arbeitskräfte bewegten (vgl. Leborgne/Lipietz 1992). Reformorientierte politische Strategien visierten demzufolge einen neuen Klassenkompromiss an, der auf die high road der kapitalistischen Rationalisierung, die Verbindung von Produktivitätssteigerungen und erweiterten Autonomiespielräumen der Lohnabhängigen zielte.

Allerdings konnte von einem „Ende der Arbeitsteilung" (Kern/Schumann 1984) schon damals keine Rede sein. Die Musterbeispiele der „Humanisierung der Arbeit" , die Volvo-Montagewerke in Kalmar und Uddevalla, 1974 und 1988 in Betrieb genommen, fielen der Krise Anfang der 1990er Jahre zum Opfer. Auch in Deutschland setzte nach der Euphorie be züglich der „Humanisierung“ der Arbeit eine gewisse Ernüchterung ein. Wenn es ein Leitbild gab, so war es Anfang der 1990er Jahre nicht der "Kalmarismus", sondern der "Toyotismus", der von US amerikanischen Forschern zum Modell der „schlanken Produktion“ verallgemeinert worden war (vgl. Womack u.a. 1990) und vor allem unter Kostensenkungsgesichts punkten angewandt wurde. Auch das Produktionssystem von Toyota stellte in mancher Hinsicht eine Abkehr vom tayloristisch-fordistischen Modell dar. Der für den Fordismus charakteristische ,gescho- bene Produktionsfluss" wurde durch einen gezogenen Produktionsfluss" ersetzt: just in-time-Produktion, vorbeugende stat nachgelagerter Qualitätskontrolle, „kontinuierlicher Verbesserungsprozess". Die kurzzyklische Arbeit am Fließsband wurde im Toyota-Produktionssystem jedoch keineswegs aufgehoben, vielmehr wurde die Arbeit weiter intensiviert. Parallel dazu war seit den 1990er Jahren auch eine Rücklkehr zu klassischen Formen des Taylorismus in der Automobilmontage zu diagnostizieren (Jürgens 1997, Springer 1999).

Die meisten Industriesoziologen wie auch ihre Kritiker konzentrierten ihr Interesse damals weiterhin vorrangig auf die Automobilindustrie, doch zeigte sich spätestens seit den 1970er Jahren, dass die Mehrzahl der Beschäftigten nicht mehr traditionell shop floor-Tätigkeiten an Drehbänken oder Fließbändern ausführte, sondern mehr und mehr Dienstleistungsarbeit leistete: sei es in den Büros der Industrieunternehmen in Rechnungswesen und Cont rolling, in Vertrieb und Marketing, in Personalwesen oder Forschung und Entwicklung, sei es in expandierenden Sektoren wie den unternehmensbezogenen oder haushaltsbezogenen Dienstleistungen. In der Tat hatten Unternehmen einen zunehmenden Bedarf an den Diensten von (gut bezahlten) Steuer- und Unternehmensberater/innen, „kreativen" Werbefachleuten oder Software-Entwicklern, aber auch (schlecht bezahlten) Call-CenterAgent/inn/en, Reinigungskräften und Wachdiensten. Demgegenüber sahen sich Haushalte oder Konsument/inn/en zwar häufig in der Situation des ,arbeitenden Kunden“, wenn frühere Bedienungsleistungen im Einzelhandel, bei Banken oder Tankstellen wegrationalisiert wurden. Aber auch für sie entstanden zahlreiche neue (oder wiederbelebte alte) Dienstleistungen vom Pizzaservice bis zur Mediation, von der Finanz- bis zur Lebensberatung, von der Tagesmutter bis zum Hundeausführdienst. Einige davon - wie die Arbeit in Call-Centern oder die "Montage" von Hamburger-Bratlingen in der Systemgastronomie - erwiesen sich als ebenso taylo- ristisch organisierbar wie Industriearbeit. Den meisten anderen fehlte dagegen das Merkmal der rigiden Standardisierung, der kurzen Taktzeiten und der strikten Vorschreibung von einzelnen Handgriffen Aber selbst wenn sie eher Züge der ,verantwortlichen Autonomie ${ }^{\text {" }}$ hatten, bedeutete dies häufig alles andere als selbstbestimmtes Arbeiten. $\mathrm{Ob}$ es sich um Angestellte in kleineren oder größeren Unternehmen, um Selbständige oder um ScheinSelbständige handelte: Sie alle gerieten direkt oder indirekt unter den Druck der shareholder value-Ökonomie und des damit verbundenen unerbittlichen Gebots des cost cutting

Die weltwirtschaftlichen Rahmenbedingungen änderten sich durch die zunehmende Globalisierung der Finanzmärkte und die wachsende Dominanz des „fiktiven Kapitals", so dass Kapitalanlagen einem verstärkten branchenübergreifenden Rendite- und Konkurrenzdruck unterworfen wurden und werden - dies betriff Stahlwerke wie Hotelketten, Autoproduzenten wie Finanzinvestitionen. Börsennotierte Alktiengesellschaften werden nunmehr tagtäglich in ihrer performance verglichen und Konzernchefs sehen ihr Prestige abhängig von den Voten der Analysten und rating-Agenturen, für die sie Quartals-Berichte vorlegen müssen. Die Zeitschrift Economist, linker Neigungen zweifellos unverdächtig, diagnostizierte bereits 1990 besorgt, der Kapitalismus habe sich weitgehend zu einem Punter's Capitalism entwickelt, also zu einem System, das nach dem Geist der Pferdewetten funktioniere: „To hold equity in a company is to own part of it... Behind the legal front, however, the functional reality of equity has been transformed. To shareholders in a typical public company in America or Britain - call it Anglo-Saxon Inc - a share is now little more than a betting slip. It is bought at what a shareholder thinks are good odds, to provide winnings that he hopes will be large. The notion that he owns part of Anglo-Saxon Inc makes as much sense to him as it would for the average gambler to imagine that he owns part of Lucky Lady, running in the 2.30 tomorrow afternoon." (May 5 1990: 8) Abhilfe gegenüber einer Orientierung, die nur noch auf kurzfristige Gewinnabschöpfung setzt, sah das Blatt in einer Wiederbelebung des Eigentümer-Unternehmers. Diesem Typus billigte das Blatt zu, auch längerfristige Ziele verfolgen zu können, bei denen eventuell mit Ressourcen pfleglicher umgegangen und Arbeitskräften Entwicklungsmöglichkeiten zugestanden wurden.

Die Erwartung nicht nur hoher, sondern stetig steigender Renditen hat unmittelbar zu einem Druck auf Löhne und Arbeitszeiten geführt. Drohten Unternehmen in den vergangenen Jahren mit Produktionsverlagerungen mit der Begründung, sie hätten sonst Verluste zu befürchten, so scheuen sie heutzutage nicht mehr davor zurück, solche Maßnahmen auch bei üppig sprudelnden Gewinnen für legitim zu erklären, wie die geplante Schließung des Nokia-Werkes in Bochum beispielhaft zeigt Die von den Finanzinvestoren erwarteten Gewinnspannen können unter der Bedingung verschärfter Konkurrenz nicht mehr per mark-up-pricing auf steigende Kosten aufgeschlagen werden. Vielmehr kalkulieren die Unternehmen heute häufig zunächst, zu welchem Preis ihr Produkt im Markt noch absetzbar ist. Von diesem Preis wird dann die erwartete Gewinnspanne abgezogen, und daraus ergeben sich die Zielkosten, die nicht überschritten werden dürfen. Durch diese Kombination der Konzepte des shareholder value und des target costing werden Löhne und Arbeitsbedingungen zur abhängigen Variable.

Dass dies durchsetzbar ist, liegt nicht zuletzt daran, dass der "stumme Zwang der ökonomischen Verhältnisse" seit der Transformation des „realexistierenden Sozialismus" in Osteuropa und Ostasien weltweit wieder sehr viel stärker zur Geltung kommt. Mit der Öffnung dieser Länder zum Weltmarkt ist das dem Kapital zur Verfügung stehende globale Heer von Arbeitskräften schlagartig angewachsen.

In den 1980er Jahren schien es zwei gegenläufige und unvereinbare Strategien der 
Arbeitsorganisation zu geben: den Neotaylorismus einerseits und die ,verantwortliche Autonomie" der Arbeitskräfte - heute als "Subjektivierung von Arbeit" (vgl. Moldaschl/Voß 2002) diskutiert - andererseits. Doch mittlerweile wurde deutlich, dass sich Momente der Subjektivierung von Arbeit und des Taylorismus bzw. de Standardisierung von Arbeit sehr woh kombinieren lassen, wie in dem Beitrag von Ingo Matuschek, Frank Kleemann und $G$. Günter Voß gezeigt wird. Demgegenüber untersuchen Nick Kratzer, Wolf gang Menz, Sarah Nies und Dieter Sauer die Folgen der beschriebenen Veränderungen auf die Leistungspolitik.

Bereits der durch Foucault inspirierte Begriff der Subjektivierung von Arbeit verweist darauf, dass es hier nicht nur bzw. nicht vorrangig um mehr Autonomie der Arbeitenden geht. Im Französischen bedeutet subjectivation auch Unterwerfung, ist also ein herrschaftskritischer Begriff. Die Autonomie der Arbeitenden bleibt auch bei der Subjektivierung von Arbeit begrenzt, weil der beschränkte Zweck der kapitalistischen Produktion, die Verwertung des Werts, in Form der indirekten Steuerung (vgl. Peters/Sauer 2005) durch Vorgabe von Renditezielen gesetzt wird. Selbst wenn der Markt im Innern der Unternehmen nur simuliert ist, bedeutet „Vermarktlichung“, dass den Beschäftigten Ziele vorgesetzt werden, die sich ständig weiter fortbewegen, je mehr sie sich ihnen annähern. Offen bleibt, wie sich die inneren Widersprüche der Subjektivierung von Arbeit artikulieren. Denn wenn die Subjektivität der Beschäftigten mobilisier wird, um die Produktivität zu steigern (oder auch, um die Arbeit zu intensivieren), kann dies durchaus mit anderen Aspekten der Nutzung der Arbeitskraft oder ihrer Ausbeutung kollidieren.

Auch im Bereich der Arbeitszeitpolitik ist es zu einem Trendbruch gekommen. Seit dem 19. Jahrhundert waren der Kampf um den "Normalarbeitstag" und die Verkürzung der Arbeitszeit nicht nur Voraussetzung für eine geregelte Reproduktion der Arbeiterklasse, sondern ebenso für die zu- nehmende Dominanz der relativen Mehrwertproduktion und den Übergang von der vorwiegend extensiven zur vorwiegend intensiven Akkumulation (vgl. Aglietta 1979). Der vorläufige Höhepunkt in dieser langfristigen historischen Bewegung wurde mit dem Kampf um die 35-Stundenwoche erreicht. Doch in den letzten Jahren hat sich dieser Trend offenbar umgekehrt. Schon die 35-Stundenwoche konnte als Norm nicht gesellschafflich verallgemeinert werden, und dort wo sie eingeführt wurde, wurde sie mit der Ausdehnung der Maschinenlaufzeiten und der Flexibilisierung der Arbeitszeiten bezahlt. Inzwischen drängt das Kapital auf eine Rückkehr zur 40-Stundenwoche, während der öffentliche Dienst Vorreiter einer Arbeitszeitverlängerung über die 40-Stundenwoche hinaus ist. Einen breiten Überblick über die Arbeitszeitentwicklung gibt Christoph Hermann, mit gewerkschaftlichen Reaktionen setzt sich der Beitrag von Sophie Jänicke, Kay Ohl und Hilde Wagner auseinander.

Aber nicht allein die Arbeitszeit wird verlängert. Hinzu kommt, dass das „Normalarbeitsverhältnis" immer mehr durch diverse Formen der „atypischen Beschäftigung" bzw. der Prekarisierung von Arbeit ausgehöhlt und verdrängt wird. Auffallend zugenommen haben in den letzten Jahren die Leiharbeit, die ungesicherte Teilzeitarbeit bzw. „geringfügige" Beschäftigung, außerdem niedrige, nicht existenzsichernde Löhne und die Zahl der "neuen“" oder "Solo-Selbständigen" (vgl. zu letzteren den Beitrag von Mario Candeias). Die geläufige Rede von den Kern- und Randbelegschaften verdeckt, dass die „Ränder“ nicht nur immer größer werden, sondern auch auf die Arbeitsbedingungen, das Bewusstsein und das Verhalten des kleiner werdenden "Kerns" der Beschäftigten im „Normalarbeitsverhältnis" abstrahlen. Die normierende Kraft des Normalarbeitsverhältnisses wird somit immer geringer. Sie macht sich inzwischen vor allem negativ bemerkbar: als Ausschluss der Prekanisierten aus den überkommenen Systemen der sozialen Sicherung, die immer noch weitgehend an das Normalarbeitsverhältnis gekoppelt sind.
Es bleiben die Fragen, wie Herrschaft unter diesen widersprüchlichen Bedingungen funktioniert, wie - jenseits des "stummen Zwangs" der ökonomischen Verhältnisse Konsens hergestellt wird, aber auch, wo Widersprüche aufbrechen und Möglichkeiten für die Artikulation von gemeinsamen Interessen derer entstehen, die sich immer mehr an den Rand gedrängt sehen. Vielfach wird der Klassenkampf gleichsam ins Innere der Individuen verlagert. So stellt Mario Canderas die Widersprüche zwischen Unternehmergeist und Prekarität dar, in denen sich die neuen „SoloSelbständigen" bewegen. Ingrid Artus zeigt in ihrem Beitrag, wie die ,repressive Integration" in die "Betriebsfamilie" auch bzw. gerade unter den Bedingungen prekärer Arbeit in multinational operierenden Dienstleistungsunternehmen mehr oder minder funktioniert. Der Kampf um eine kollektive Interessenvertretung erscheint unter diesen Bedingungen als, verrückt ${ }^{\text {“ }}$ und findet dennoch manchmal mit Erfolg statt.

Nicht unwesentlich verschieden sind auch die gewerkschaftlichen Handlungsbedingungen in Ostdeutschland. Zu lange wurde hier versucht scheinbar erfolgreiche Muster gewerkschaftlicher Arbeit einfach $\mathrm{zu}$ übertragen. Demgegenüber plädiert Robert Hinke dafür die neuen Bundesländer als eigensinniges Handlungsterrain anzuerkennen.

Die gegenwärtigen Kämpfe in und um $\mathrm{Ar}$ beit sind in einem hohen Maße politisiert. Beispiethaft dafür sind die Auseinandersetzungen um einen Mindestlohn, um neue Formen der sozialstaatlichen Absicherung angesichts brüchiger und prekärer Erwerbsbiographien oder auch um die europäische Dienstleistungsrichtlinie. Damit wird die Frage nach gesellschaftlichen Interessenkoalitionen zentral. Die tiefgreifenden Veränderungen in den Arbeits- und Beschäftigungsverhältnissen haben die gesellschaftlichen Ungleichheiten neu konturiert. Robert Castel hat dafür das Bild der drei "Zonen " vorgeschlagen, die sich nach dem Grad der Stabilität und Qualität des Zugangs zur Erwerbsarbeit, der sozialstaat- lichen Absicherungen und der Reichweite und Tragfähigkeit sozialer Einbindungen unterscheiden: die Zonen der „Integration $^{\text {"c }}$, der "Verwundbarkeit" und der ,Abkoppelung" oder sozialen Ausgrenzung. Die Erfahrungen zunehmender Intensivierung und Unsicherheit der Erwerbsarbeit, von fremdbestimmter Flexibilität, Abstiegsängsten und sozialer Ungerechtigkeit reichen gegenwärtig bis weit in die "Zone der Integration" hinein, obwohl dort tarifvertragliche Regelungen noch Bestand haben und sozialstaatliche Absicherungen der Erwerbsposition bislang noch greifen. Entscheidend wird daher sein, inwieweit es gelingt, um die Themen der Verbindung von Sicherheit und Autonomie in und jenseits der Erwerbsarbeit Interessenkoalitionen zwischen den Lohnabhängigen der verschiedenen "Zonen" zu schaffen. Sonst drohen Entsolidarisierung und weitere Niederlagen bei den Kämpfen in der und um die Arbeit.

Umkämpfie Arbeit konnte in diesem Heft nur schlaglichtartig beleuchtet werden wir verstehen dies als den Beginn einer aktuellen Bestandsaufnahme bzw. als Moment einer fortlaufenden Beschäftigung mit dem Thema, die die PROKLA von Beginn an gekennzeichnet hat. Daher laden wir dazu ein, uns auch in Zukunft Manuskripte zu diesem Themenkreis zuzusenden, und zwar keineswegs nur bezogen auf Arbeit in Deutschland. Industriesoziologische und arbeitspolitische Analysen sollten unserer Ansicht nach wieder stärker in den Kontext der Kritik der politischen Ökonomie bzw. kritischer Gesellschaftstheorie gestellt werden, als es in den letzten Jahren üblich war.

$$
* * *
$$

Gegenwärtig sind die US-amerikanische Immobilienkrise und ihre Auswirkungen auf das internationale Finanzsystem in aller Munde. Genau vor einem Jahr veröffentlichten wir in PROKLA 146 (März 2006) „Das Ende der Immobilienblase in den USA", einen Artikel von Trevor Evans, der auch heute noch lesenswert ist. Mit dem Finanzsystem und der Rolle von 
Finanzinvestoren beim Umbau der deutschen Wirtschaft beschäftigt sich in diesem Heft (außerhalb des Schwerpunkts) der Artikel von Martin Beckmann.

Zwei Artikel in den beiden letzten Nummern der PROKLA regten zum Widerspruch an. Raimund Feld wirft einen kritischen Blick auf die Analyse der Präsidentschaftswahlen von Kolja Lindner in PROKLA 148 (September 2007) und Klaus Lederer antwortet auf Henrik Lebuhns Untersuchung der Berliner Stadtpolitik und der Rolle der Linkspartei in PROKLA 149 (Dezember 2007). In kurzen Beiträgen setzten sich die beiden kritisierten Autoren mit ihren Kritikern auseinander.

\section{Literatur}

Aglietta, Michel (1979): A theory of capitalist regulation. London.

Jürgens, Ulrich (1997): Rolling back cycle times: The renaissance of the classical assembly line in final assembly. In: Koichi Shimokawa u.a.
(Hg.), Transforming automobile assembly, Berlin u.a., 255-273.

Kern, Horst; Schumann, Michael (1984): Das Ende der Arbeitsteilung? Rationalisierung in der industriellen Produktion: Bestandsaufnahme, Trendbestimmung. München.

Leborgne, Danièle; Lipietz, Alain (1992): Conceptual fallacies and open questions on PostFordism. In: Michael Storper. Allen J Scott $(\mathrm{Hg})$, Pathways to industrialization a cott (Hg.), Pathways to industrialization and re332-348. Moldaschl, Manfred; Voß, G. Günter (Hg.) (2002): Subjektivierung von Arbeit. München, Mering.

Peters, Klaus; Sauer, Dieter (2005): Indirekte Steuerung - eine neue Horrschaftom Hilde Wagner ( $\mathrm{Hg}$ ) Rentier' ' I noch?" Noue Steles), "Renties ich mich noch? Neue Steuerungskonzepte im Betrieb, Hamburg, 23-58.

Springer, Roland (1999): Rückkehr zum Taylorismus? Arbeitspolitik in der Automobilin dustrie an Scheideweg. Frankfurt, New York.

Womack, James P.; Jones, Daniel T. Roos, Daniel (1990): The machine that changed the world. New York.

\section{PROKLA 151 (Juni 2008): Gesellschaftstheorie nach Marx
und Foucault}

Nachdem in den 1960er und 70er Jahren kritische Gesellschaftstheorien vor allem im Anschluss an Marx formuliert wurden, ist in den 1980er und 90er Jahren Foucault zu einem entscheidenden Stichwortgeber geworden. Dabei blieb das Verhältnis von Marx und Foucault allerdings recht unklar: Foucault, der sich selbst keineswegs als Marxist verstand, knüpfte in wichtigen Feldern an Marx an, wurde in der Debatte aber auch immer wieder gegen Marx in Stellung and wurde in Formation der "Diskurse“ gegen die "Magialien se stehen oder deren Bestandteil sei, blieb genauso unklar wie das Verhältnis der Foucaultschen „Mikrophysik der Macht“ zum Marxschen Begriff von Hertschaft und Ausbeutung. Allerdings soll es in diesem Heft nicht begriff von Herrschaft Aufarbeitungen gehen, vielmehr Anschluss an Marx lysiert und der Widerand ysiert und der Widerstand dagegen konzipiert werden kann.
Nick Kratzer, Wolfgang Menz, Sarah Nies, Dieter Sauer

\section{Leistungspolitik als Feld "umkämpfter Arbeit"}

In den 1990er Jahren des letzten Jahrhunderts ist die Krise des Fordismus in eine neue Phase eingetreten - und mit ihr auch die Kämpfe und Konflikte um Arbeit. Während in den 1970er Jahren gewissermaßen die Krise entdeckt wird, zeichnen sich die 1980er Jahre vor allem durch Suchprozesse aus, neue Konzepte werden lediglich partiell realisiert. Erst in den 1990er Jahren beginnen sich neue Rationalisierungsleitbilder und -konzepte flächendeckend durchzusetzen und zu einem neuartigen Übergangsregime zu verdichten, dessen Kern eine marktzentrierte Produktionsweise bildet (vgl. Dörre 2001; Sauer 2005a). Die Herausbildung eines neuen, marktzentrierten Rationalisierungsregimes lässt sich als Ausdruck eines tiefgehenden gesellschaftlichen Umbruchprozesses interpretieren. Wir befassen uns im Folgenden mit denjenigen Konfliktfeldern von Arbeit, die vor dem Hintergrund dieses Umbruchprozesses von Bedeutung sind. Vor allem lenken wir unser Augenmerk auf einen Politikbereich, der gerade nicht umkämpft zu sein scheint, jedenfalls nicht im Sinne manifester Auseinandersetzungen auf betrieblicher oder kollektivvertraglicher Ebene: das Feld der Arbeits- bzw. konkreter: der Leistungspolitik.

Im Mittelpunkt unserer Überlegungen stehen drei Entwicklungstendenzen: Prekarisierung, Subjektivierung und Standardisierung - sowie ihre Folgen für betriebliche Arbeits- und Leistungspolitik. Diese Entwicklungstendenzen sind in der gegenwärtigen Debatte mehr als nur unterschiedliche Perspektiven auf den Umbruch in der Entwicklung von Arbeit. Sie sind oft konkurrierende Deutungsangebote für zentrale Entwicklungstendenzen - und damit auch für die arbeitspolitischen Herausforderungen und Konfliktfelder: Angesichts der Kämpfe um Beschäftigungs- und Entgeltsicherung erscheinen leistungspolitische Fragestellungen nicht Wenigen als Luxusfragen, und neue Steuerungsformen von Arbeit, die auf die erweiterte „Inbetriebnahme“ des Subjekts setzen, scheinen durch eine Renaissance des Taylorismus konterkariert zu werden oder nach dem Ende der New Economy überholt zu sein.

Wenn wir hier die betriebliche Leistungspolitik als Feld (noch nicht) „um kämpfter Arbeit" ins Zentrum stellen, dann geht es uns auch um die Überwindung solcher nicht nur oft fruchtlosen, sondern auch falschen Gegenüberstellungen. Der gegenwärtige Umbruchprozess besteht nicht zuletzt darin, dass unter dem Druck einer radikalisierten Marktökonomie die Klaviatur betriebli- 